ARTICLE

DOI: $10.1038 /$ s41467-017-00404-y

\title{
Phytochrome-interacting factors directly suppress MIR156 expression to enhance shade-avoidance syndrome in Arabidopsis
}

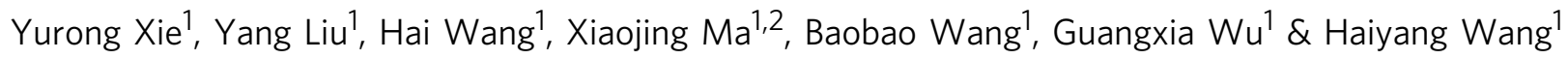

\begin{abstract}
Plants have evolved a repertoire of strategies collectively termed the shade-avoidance syndrome to avoid shade from canopy and compete for light with their neighbors. However, the signaling mechanism governing the adaptive changes of adult plant architecture to shade is not well understood. Here, we show that in Arabidopsis, compared with the wild type, several PHYTOCHROME-INTERACTING FACTORS (PIFS) overexpressors all display constitutive shade-avoidance syndrome under normal high red to far-red light ratio conditions but are less sensitive to the simulated shade, whereas the MIR156 overexpressors exhibit an opposite phenotype. The simulated shade induces rapid accumulation of PIF proteins, reduced expression of multiple MIR156 genes, and concomitant elevated expression of the SQUAMOSA-PROMOTER BINDING PROTEIN-LIKE (SPL) family genes. Moreover, in vivo and in vitro assays indicate that PIFs bind to the promoters of several MIR156 genes directly and repress their expression. Our results establish a direct functional link between the phytochrome-PIFs and miR156-SPL regulatory modules in mediating shade-avoidance syndrome.
\end{abstract}

\footnotetext{
${ }^{1}$ Biotechnology Research Institute, Chinese Academy of Agricultural Sciences, Beijing 100081, China. ${ }^{2}$ Graduate School of Chinese Academy of Agricultural Sciences, Beijing 100081, China. Yurong Xie and Yang Liu contributed equally to this work. Correspondence and requests for materials should be addressed to H.W. (email: wanghaiyang@caas.cn)
} 
D the year 2050, the world population is expected to reach 9.3 billion and it is a daunting task to produce enough food, feed, and biofuel materials with limited resources ${ }^{1}$. Over the past few decades, increasing planting density has been an effective way of improving crop yields per unit land $\mathrm{area}^{2}$. However, a key factor that limits planting density in modern agricultural practice is the plant's shade-avoidance response, which is triggered when plants detect a reduction of red (R) to far-red (FR) light ratios (R: $\mathrm{FR}$ ) in their environment due to absorption of $\mathrm{R}$ light by neighboring plants. Typical shade-avoidance responses include increased plant height, elevated leaf angles to horizontal, reduced branching, decreased leaf blade area, and early flowering ${ }^{3}$. These traits are collectively called shade-avoidance syndrome (SAS) ${ }^{4}$. Although it is deemed that a robust SAS allows the plants to compete with the neighbors for limited resources, and to complete their life cycle earlier to ensure a reproductive success such plasticity comes at a cost of reduced fitness ${ }^{5}$. For crop species, the SAS could cause a reduction in yields due to reduced investment of resources on reproductive development and decreased immunity to plant pests and pathogens ${ }^{6,7}$. Thus, it is generally believed that SAS has largely been attenuated or refined during crop domestication and genetic improvement ${ }^{8}$. However, currently little is known regarding the molecular genetic mechanisms governing SAS in crops, which severely hampers our ability to breed high-density tolerant crop cultivars.

In the model dicot plant species Arabidopsis thaliana, SAS is primarily mediated by the photoreceptor phytochrome B (phyB), with phyD and phyE playing a minor role ${ }^{9}$. Arabidopsis phyB mutants exhibit a constitutive shade-avoidance response even under normal high R:FR conditions, including elongation of hypocotyl, petioles and stem, accelerated flowering, and increased apical dominance, indicating that phyB negatively regulates
SAS $^{10}$. Phytochrome is synthesized within the cytosol in its inactive $\mathrm{R}$ light absorbing Pr form, and upon exposure to $\mathrm{R}$ light it can rapidly convert into its biologically active FR lightabsorbing Pfr form. Reversion of Pfr to Pr form occurs in FR light-enriched environments or more slowly in the dark ${ }^{11}$. Thus, under shade conditions (low R:FR), the majority of phyB is inactivated, thus triggering a shade-avoidance response.

Previous studies have also identified numerous regulators of SAS acting downstream of the phytochrome photoreceptors in Arabidopsis. Among them, a family of PHYTOCHROMEINTERACTING FACTORS (PIFs, including PIF1, PIF3, PIF4, PIF5, and PIF7) is believed to play an instrumental role in mediating shade-induced rapid transcriptome reprogramming and subsequent SAS responses ${ }^{12-15}$. Among the PIFs-regulated genes, a dozen of PHYTOCHROME RAPIDLY REGULATED (PAR) genes (e.g., ATHB2, ATHB4, HFR1, PAR1, PAR2, PIL1, HAT1, HAT2, HAT3, BEE1, BIM1, BBX21, BBX22, BBX24, and $B B X 25)$ are believed to be essential for implementing the SAS responses. These factors either act positively or negatively to bring about a balanced SAS responses ${ }^{16}$. In addition, it has been shown that several PIFs (PIF4, PIF5, and PIF7) can directly regulate the expression of several auxin biosynthetic (TAA1 and YUC genes) or response genes (such as IAA29) to promote hypocotyl elongation ${ }^{13}, 15,17$. However, it should be noted that most studies on SAS responses have been focused on the hypocotyl elongation process in Arabidopsis. A recent study combining RNA-seq with phenotypic profiling revealed that each shadeavoidance response (hypocotyl, petiole, and flowering time) was governed by shared and separate pathways, suggesting that cautions should be excised when generalizing conclusions from studies on hypocotyls ${ }^{18}$. Thus, our understanding of SAS molecular mechanisms remains incomplete and fragmented.

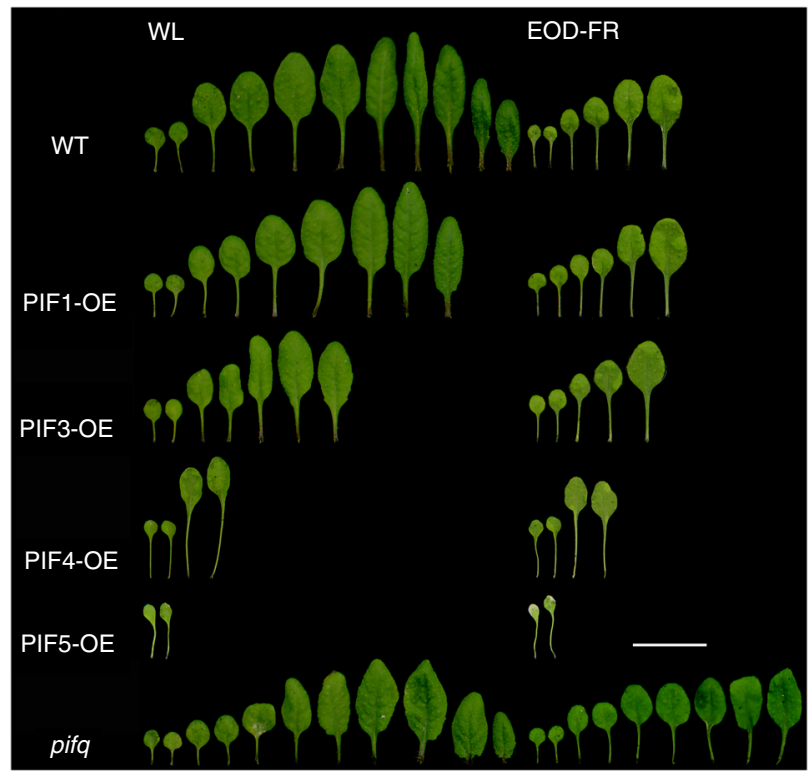

b
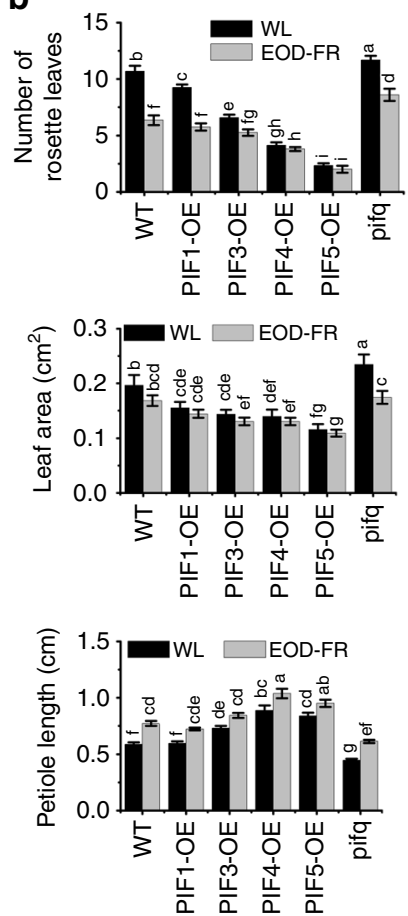

Fig. 1 Adult PIF overexpressors exhibit constitutive SAS under normal high R:FR conditions and attenuated SAS responses under simulated shade conditions. a Comparison of the rosette leaf number, leaf blade area, and petiole length of the first and second leaf between PIF overexpressors, pifa, and WT plants grown under normal high R:FR (WL) or simulated shade (EOD-FR) conditions. Eight-day-old seedlings were moved into the soil and grown under WL with or without EOD-FR treatment for 4 weeks. Bar $=2 \mathrm{~cm}$. $\mathbf{b}$ Quantification of the rosette leaf number, leaf blade area, and petiole length of PIF ovexpressors, pifq, and WT under WL or EOD-FR conditions. Values shown are mean \pm SD $(n=12)$. Different letters indicate significant differences by twoway ANOVA 

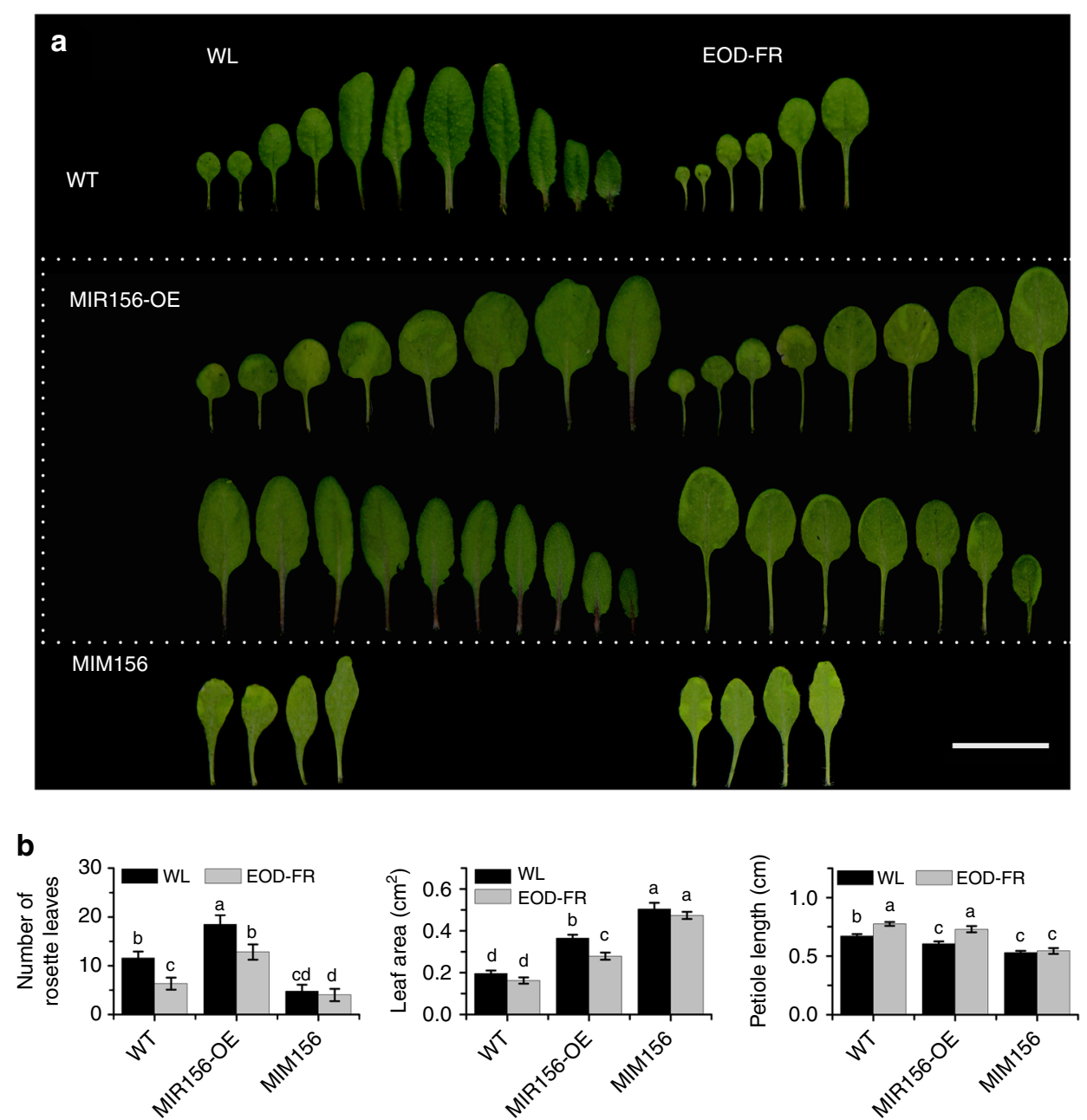

Fig. 2 MIR156 overexpression represses attenuated SAS responses in adult Arabidopsis plants. a Comparison of the rosette leaf number, leaf blade area, and petiole length of the first and second leaf between MIR156overexpessors, MIM156, and WT plants under normal high R:FR (WL) or simulated shade (EODFR) conditions. Eight-day-old seedlings were moved into the soil and grown under WL with or without EOD-FR treatment for 4 weeks. Bar $=2 \mathrm{~cm}$. b Quantification of the rosette leaf number, leaf blade area, and petiole length of MIR156 overexpressors, MIM156, and WT plants under WL or EOD-FR conditions. Values shown are mean $\pm \mathrm{SD}(n=12)$. Letters indicate significant differences by two-way ANOVA

Recent studies have shown that MIR156s, together with their downstream targets SQUAMOSA-PROMOTER BINDING PROTEIN-LIKE (SPL) family of genes, are pivotal regulators of various biological processes in plants, such as the timing of vegetative to reproductive phase transition, leaf development, branching/ tillering, fruit ripening, fertility, and response to stresses ${ }^{19,20}$. As many of the MIR156s-mediated developmental processes overlap with the phytochrome-mediated SAS responses, we investigated the regulatory relationship between the phy-PIFs signaling pathway and the miR156-SPL regulatory module in mediating SAS. We show that several PIF overexpressors (35S::PIF1-OE, 35S::PIF3-OE, 35S::PIF4-OE, and 35S::PIF5-OE) all display enhanced SAS responses under normal high R:FR ratio conditions, and attenuated sensitivity to the simulated shade (including leaf number, leaf blade size, branches, plant height, petiole length, and flowering time), compared to the wild-type plants (WT). In addition, the accumulation of PIF proteins rapidly increases in response to simulated shade. Moreover, multiple PIFs (PIF1, PIF3, PIF4, and PIF5) can directly bind to the G-box motifs present in the promoters of several MIR156 genes and downregulate their expression. Our results provide a direct functional link between the phytochrome-PIFs and miR156-SPL regulatory modules in mediating SAS during plant vegetative growth and development.

\section{Results}

Adult PIF overexpressors exhibit constitutive SAS. Previous studies showed that at the seedling stage, the shade-induced hypocotyl elongation is significantly compromised in the pif4 pif5 double mutant, and to an even greater extent in the pif1 pif3 pif4 pif5 quadruple (pifq) and pif7 mutants ${ }^{12}, 14,15$. Conversely, seedlings overexpressing PIF4 and PIF5 have constitutively long hypocotyls and petioles even under normal high R:FR conditions $^{12}$. To examine the effects of PIF overexpression at the vegetative and adult stages, we obtained the previously generated 35S::PIF1-OE, 35S::PIF3-OE, 35S::PIF4-OE, and 35S::PIF5-OE transgenic plants ${ }^{21-24}$, together with the WT control and pifq mutant, and grew them under normal high R:FR conditions. The blade area of the first and second leaf, length of the first and second petiole, number of rosette-leaf branches, number of rosette leaves at bolting, and plant height were measured. Our data showed that under high R:FR conditions, the numbers of both rosette leaves and rosette-leaf branches were reduced significantly in the PIF overexpressors, especially in the 35S::PIF4$O E$ and 35S::PIF5-OE lines. On average, the 35S::PIF-OE lines had only $\sim 21.8-86.5 \%$ rosette leaves and $\sim 8.6-76.4 \%$ rosette-leaf branches, respectively, compared with the WT (Fig. 1a, b and Supplementary Fig. 1a, b). Also, the first and second petioles of the 35S::PIF-OE lines were much longer ( 24.1-52.3\% longer 
a
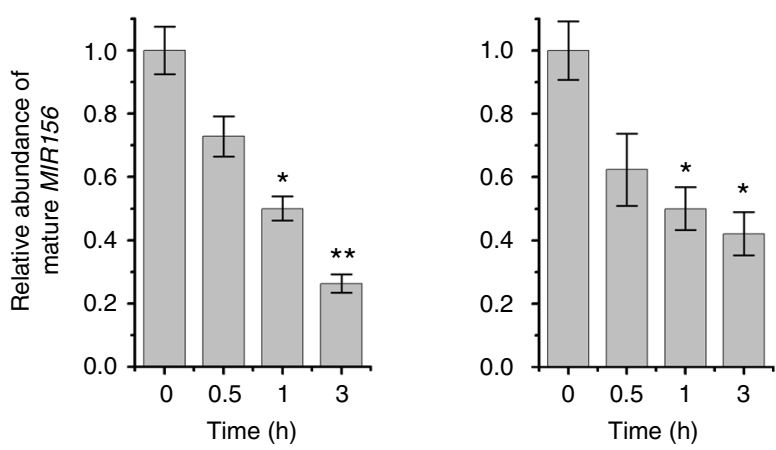

b
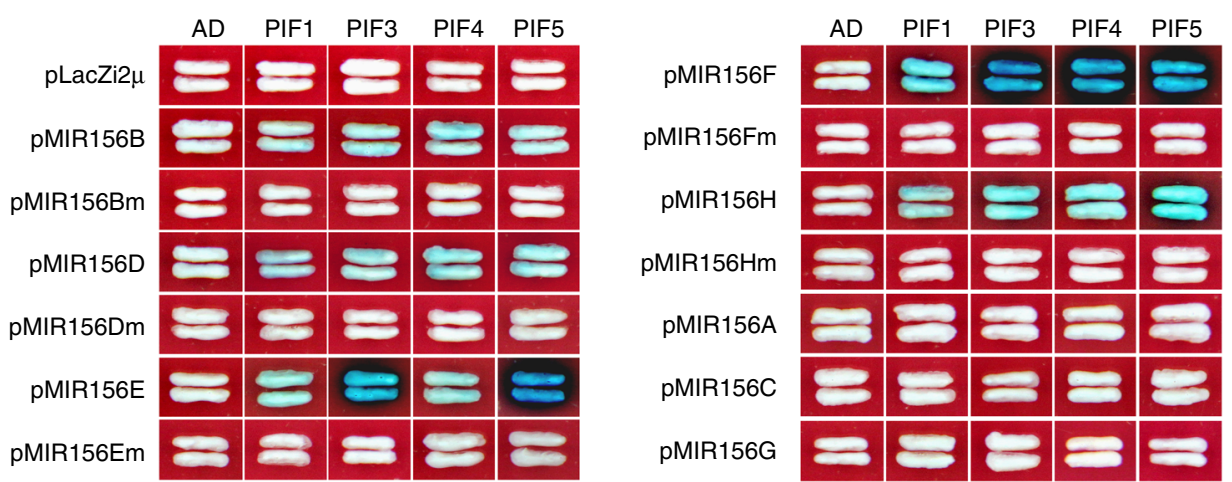

C
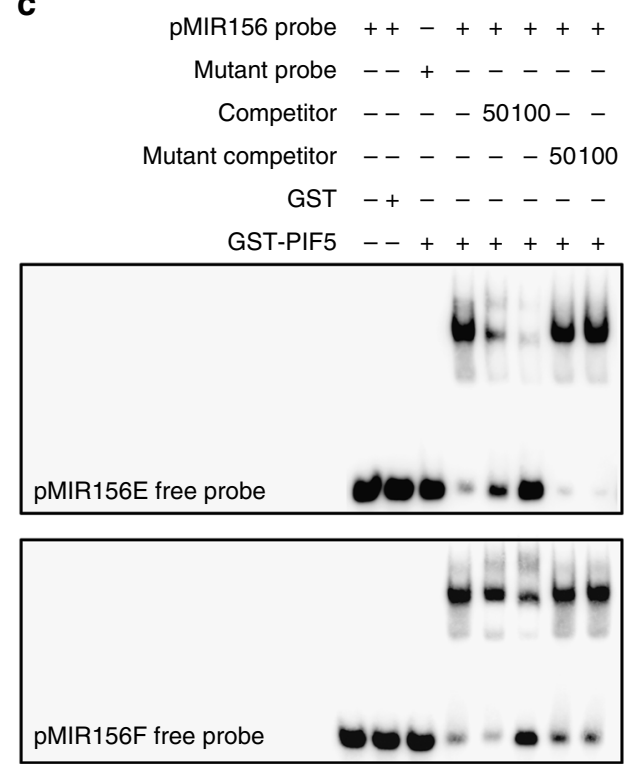

d

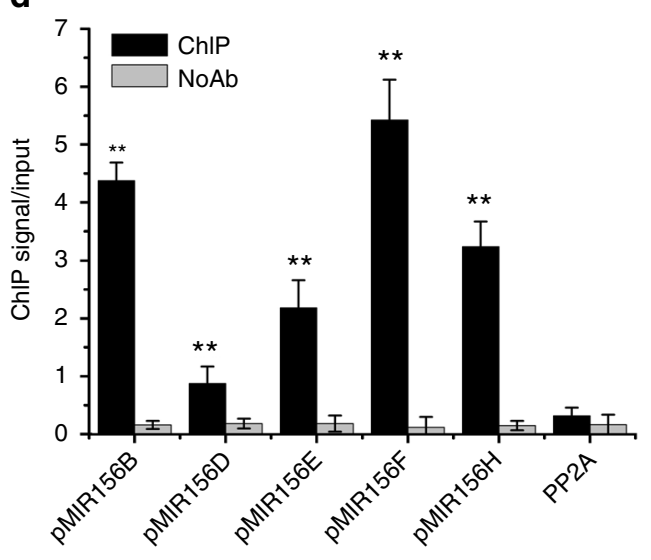

Fig. 3 PIFs directly bind to MIR156 promoters. a The transcript levels of mature MIR156 decline in WT in response to EOD-FR treatment. Eight-day-old (left panel) or 3-week-old (right panel) WT plants grown under WL were treated with or without EOD-FR for 6 days before harvested for RNA extraction. Values given are mean $\pm \mathrm{SD}(n=3) .{ }^{\star} P<0.05$ and ${ }^{\star \star} P<0.01$ by the Student's $t$-test. $\mathbf{b}$ Y1H assay showing direct binding of PIFs to the PBE-box or G-box motifs in several MIR156 promoters. The length and PIF-binding elements of each promoter fragment is shown in Supplementary Fig. 6 . c EMSA showing that the GST-PIF5 bHLH domain recombinant protein binds to biotin-labeled probes of MIR156E (up panel) and MIR156F (down panel). d ChIP assay of MIR156 in 2week-old 35S::PIF5-HA seedlings grown under high R:FR conditions and treated with EOD-FR for one time before harvesting. Values given are mean \pm SD $(n=3) .{ }^{\star} P<0.01$ by the Student's $t$-test

than that of WT). In addition, the blade sizes of the first and second leaf were significantly smaller in the $35 S:: P I F-O E$ lines compared with the WT ( 59.1-78.8\% of that of WT) (Fig. 1a, b). At maturity, all 35S::PIF-OE lines (except PIF1-OE line) were taller than the WT and all 35S::PIF-OE lines flowered earlier than WT when measured by the number of rosette leaf at bolting
(Supplementary Fig. 1a, b). In contrast, the pifq mutant displayed a largely opposite phenotype to the 35S::PIF-OE lines (had slightly more rosette leaves, larger first and second leaves, shorter first and second petioles) (Fig. 1a, b). These observations indicate that these PIFs play a positive role in regulating various aspects of SAS at the vegetative and adult stages. 
Adult PIF overexpressors are less sensitive to shade. Since FR light treatment at the end-of-day (EOD-FR) causes similar plant phenotypic changes to those grown under canopy shade (reduced R:FR ratios $)^{4}$, thus EOD-FR treatment was adopted in this study to investigate the response of PIF-related materials to mimic shade conditions. The 35S::PIF-OE lines, pifq mutant, and WT plants were first germinated and grown under normal high R:FR (white light (WL)) conditions for 1 week, and then treated with EOD-FR for 15 min each day before returning to darkness. After EOD-FR treatment for 4 weeks, the number of rosette leaves and rosette-leaf branches, blade sizes of the first and second leaf, and lengths of the first and second petiole were measured. Compared to their counterparts grown under normal R:FR conditions, EODFR-treated WT, pifq, 35S::PIF1-OE, and 35S::PIF3-OE plants all had significantly reduced rosette leaf number, while the rosette leaf number of 35S::PIF4-OE and 35S::PIF5-OE declined slightly (Fig. 1b). Also the number of rosette-leaf branches and the blade areas of the first and second leaf were reduced, but the lengths of the first and second petioles were increased in the EOD-FRtreated plants (Fig. 1b and Supplementary Fig. 1b). Notably, under EOD-FR treatment, the 35S::PIF-OE plants had milder reductions (percentages) in leaf number, rosette leaf branches, the first and second leaf size, and only moderate increases in petiole length and plant height compared with the WT plants. In contrast, pifq plants had larger reductions (percentages) in rosette leaf branches and the first and second leaf size, and larger increases in petiole length and plant height under EOD-FR treatment compared with the WT (Fig. 1b and Supplementary Fig. 1b). These observations suggest that the 35S::PIF-OE lines were less sensitive, whereas the pifq mutant was more sensitive to the EOD-FR treatment compared with the WT.

MIR156 overexpressors exhibit attenuated SAS responses. Arabidopsis MIR156B overexpressing plants were reported to have more rosette leaves, increased branching (including axillary, quaternary, and quinary branches), prolonged expression of juvenile vegetative traits, and late flowering when grown under normal high R:FR conditions ${ }^{25,26}$. To investigate a possible role of MIR156s in shade-avoidance response, we generated overexpressors for several MIR156 genes (including MIR156B, MIR156D, MIR156E, MIR156F, and MIR156H) and they all showed similar phenotypes including more rosette leaves and late flowering (Supplementary Fig. 2a, b). In this study, MIR156B overexpressor (designated MIR156-OE hereafter) was used for further detailed investigation. When grown under our normal high R:FR conditions, MIR156-OE produced $\sim 59.6 \%$ more rosette leaves, $\sim 35.2 \%$ more rosette-leaf branches, and $\sim 86.4 \%$ bigger leaves, but obviously shorter height (only $51.3 \%$ of WT) and slightly shorter petioles ( $89.9 \%$ of WT) compared with the WT (Fig. 2a, b and Supplementary Fig. 3a, b). On the contrary, the MIM156 transgenic line, which has significantly reduced MIR156 expression $^{27}$, displayed a largely opposite phenotype: fewer rosette leaves ( $\sim 1.2 \%$ of WT) and rosette-leaf branches ( $68.5 \%$ of WT), significantly shorter petioles ( 78.6\% of WT) and much bigger first and second leaves ( 157.4\% of WT) (Fig. 2a, b and Supplementary Fig. 3a, b).

To test the role of MIR156s on SAS, both MIR156-OE and MIM156 lines were treated with EOD-FR. The results showed that EOD-FR treatment significantly reduced the rosette leaf number in both WT and MIR156-OE line ( 45.4 and $~ 30.76 \%$ reduction for WT and MIR156-OE, respectively). EOD-FR treatment also lead to reduced rosette-leaf branches $(\sim 54.9$ and $\sim 57.8 \%$ reduction for WT and MIR156-OE, respectively), smaller blade area for the first and second leaf ( 17.3 and $23.7 \%$ reduction for WT and MIR156-OE, respectively) compared with their respective counterparts grown under high R:FR (Fig. $2 \mathrm{~b}$ and Supplementary Fig. 3b). The MIR156-OE line also had greater increase in plant height ( 26.1 and $\sim 41.1 \%$ for WT and MIR156$O E$, respectively) and the first and second petiole length $(\sim 15.2$ and $\sim 21.6 \%$ for WT and MIR156-OE, respectively) in response to the EOD-FR treatment compared to the WT (Fig. $2 \mathrm{~b}$ and Supplementary Fig. 3b). Together, these results suggest that the MIR156 overexpressors exhibit reduced SAS under normal high $\mathrm{R}: F R$ ratio conditions, and are more sensitive to the simulated shade.

The transcript levels of MIR156s decrease under shade. Previous studies have shown that under high R:FR conditions, PIF1, PIF3, PIF4, PIF5, and PIF7 proteins all physically interact with phyB through their conserved N-terminal sequence, which in turn led to rapid phosphorylation and subsequent degradation of these PIF proteins by the $26 \mathrm{~S}$ ubiquitin-proteasome pathway. This provides an elegant mechanism to rapidly regulate nuclear gene expression in response to the changing light environment ${ }^{28}$. In accordance with this model, PIF3, PIF4, and PIF5 proteins accumulate rapidly upon exposure to low R:FR ${ }^{12}, 14$. To test the effect of simulated shade on PIF protein accumulation, we conducted a time course immunoblot analysis of PIF with 2-weeksold 35S::PIF1-OE, 35S::PIF3-OE, 35S::PIF4-OE and 35S::PIF5-OE seedlings exposed to EOD-FR treatment. We observed an increased accumulation of these PIF proteins in seedlings treated with EOD-FR for $30 \mathrm{~min}, 1 \mathrm{~h}$, and $3 \mathrm{~h}$ after EOD-FR treatment, compared to seedlings grown under normal WL conditions (Supplementary Fig. 4a). Quantitative reverse transcriptase (RT)PCR assay showed that expression of several shade-avoidance marker genes (PIL1, HFR1, ATHB2, CKX5, XTR7, and IAA19) ${ }^{14}$, 29 was obviously increased in PIF overexpressors (Supplementary Fig. 4b) and significantly upregulated by the EOD-FR treatment (Supplementary Fig. 4c), thus validating the effectiveness of our EOD-FR treatment.

We next examined the mature MIR156 RNA levels in plants treated with simulated shade. Quantitative RT-PCR analysis showed that the total mature MIR156 RNA level was rapidly and significantly downregulated in 2-weeks-old Arabidopsis seedlings or 4-weeks-old adult plants treated with EOD-FR (Fig. 3a). To confirm this, we examined the primary transcripts of $M I R 156 B$, MIR156D, MIR156E, MIR156F, and MIR156H in response to EOD-FR treatment. As expected, the primary transcript levels of these MIR156s all declined significantly when exposed to EODFR (Supplementary Fig. 5a). To further verify this, we generated transgenic plants harboring the $\beta$-glucuronidase (GUS) reporter gene driven by the MIR156B, MIR156D, MIR156E, MIR156F, and MIR156H promoter, respectively. The pMIR156::GUS reporter gene activities were all similarly downregulated by exposure to EOD-FR (Supplementary Fig. 5b, c). In addition, we detected the expression of several miR156-targeted SPL genes and found most of them were obviously upregulated when exposed to EOD-FR (Supplementary Fig. 5d).

PIFs directly bind to G-box motifs in MIR156 promoters. PIFs and MIR156s are positive and negative regulators of SAS, respectively, and the abundance of MIR156 decreases upon PIF protein accumulation, suggesting that PIFs may directly suppress MIR156s expression. To test this hypothesis, we first analyzed the cis-elements of the putative promoters $(\sim 3 \mathrm{~kb}$ upstream of the mature MIR156 sequence) of all eight Arabidopsis MIR156 members $(M I R 156 A-H)$ for PIF-binding sites. One or multiple typical PIF-binding sites (G-box or PBE-box) were found in all MIR156 promoters (Supplementary Fig. 6). Yeast one-hybrid assay showed that all four PIFs tested here (PIF1, PIF3, PIF4, and 
PIF5) could bind to the G-box motifs present in the promoters of MIR156B, MIR156D, MIR156E, MIR156F, and MIR156H, with PIF3 and PIF5 showing the strongest binding to the MIR156E and MIR156F promoters. Mutations of the G-box sequences in their promoters abolished the binding, suggesting that the binding was specific. No obvious binding of PIFs to the promoters of
MIR156A, MIR156C, or MIR156G was detected in this assay (Fig. 3b and Supplementary Fig. 7). To confirm this binding, we generated recombinant protein of the bHLH DNA-binding domain of PIF5 (Supplementary Fig. 8) and used it in a gel electrophoresis mobility shift assay (EMSA) with the MIR156E and MIR156F promoter fragments containing the G-box. As

a
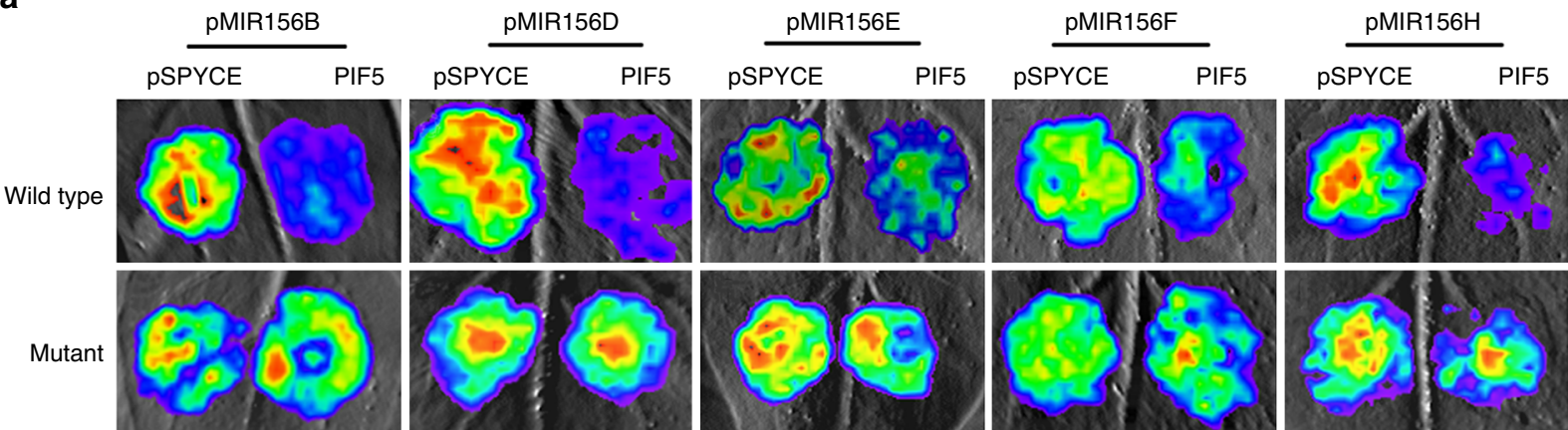

b
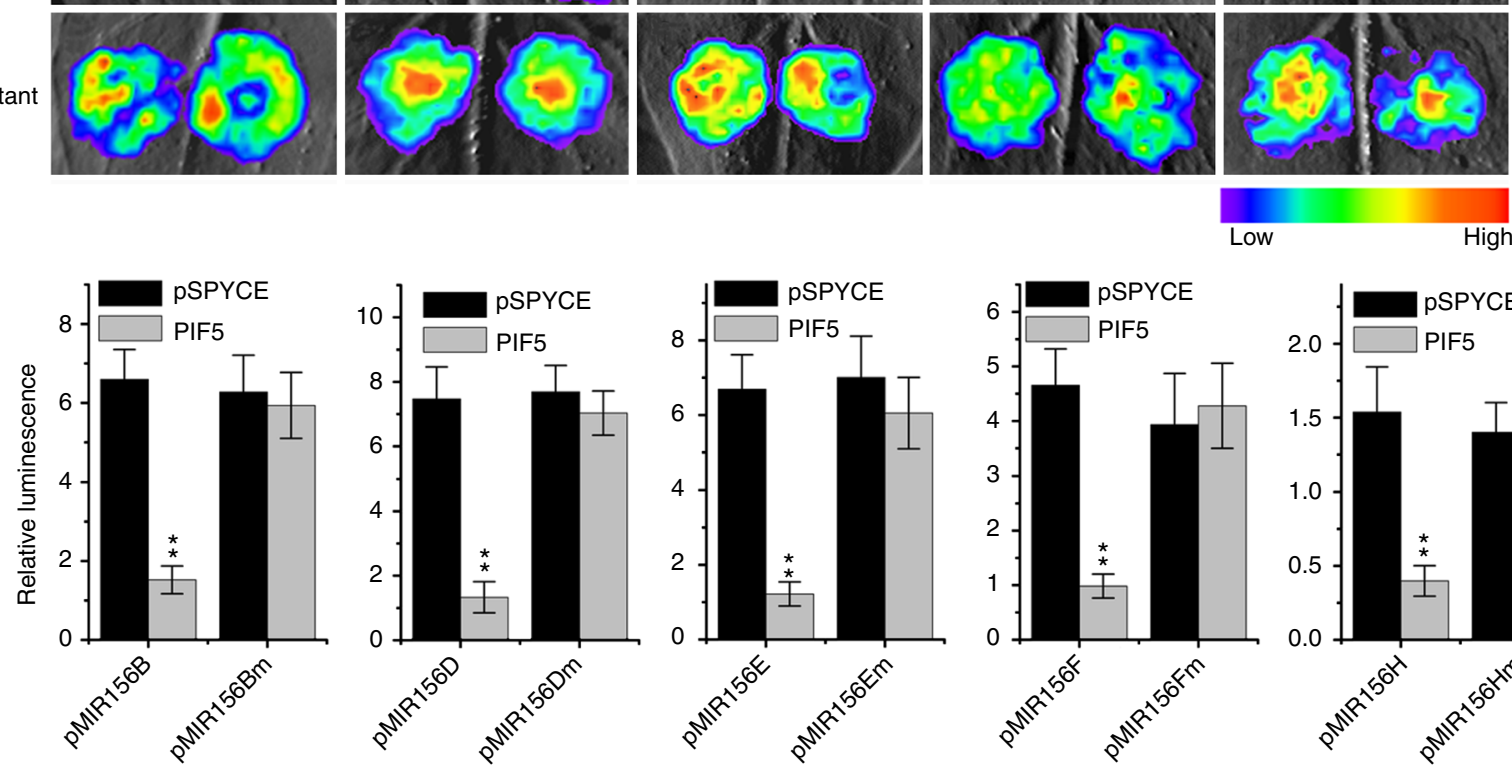

C
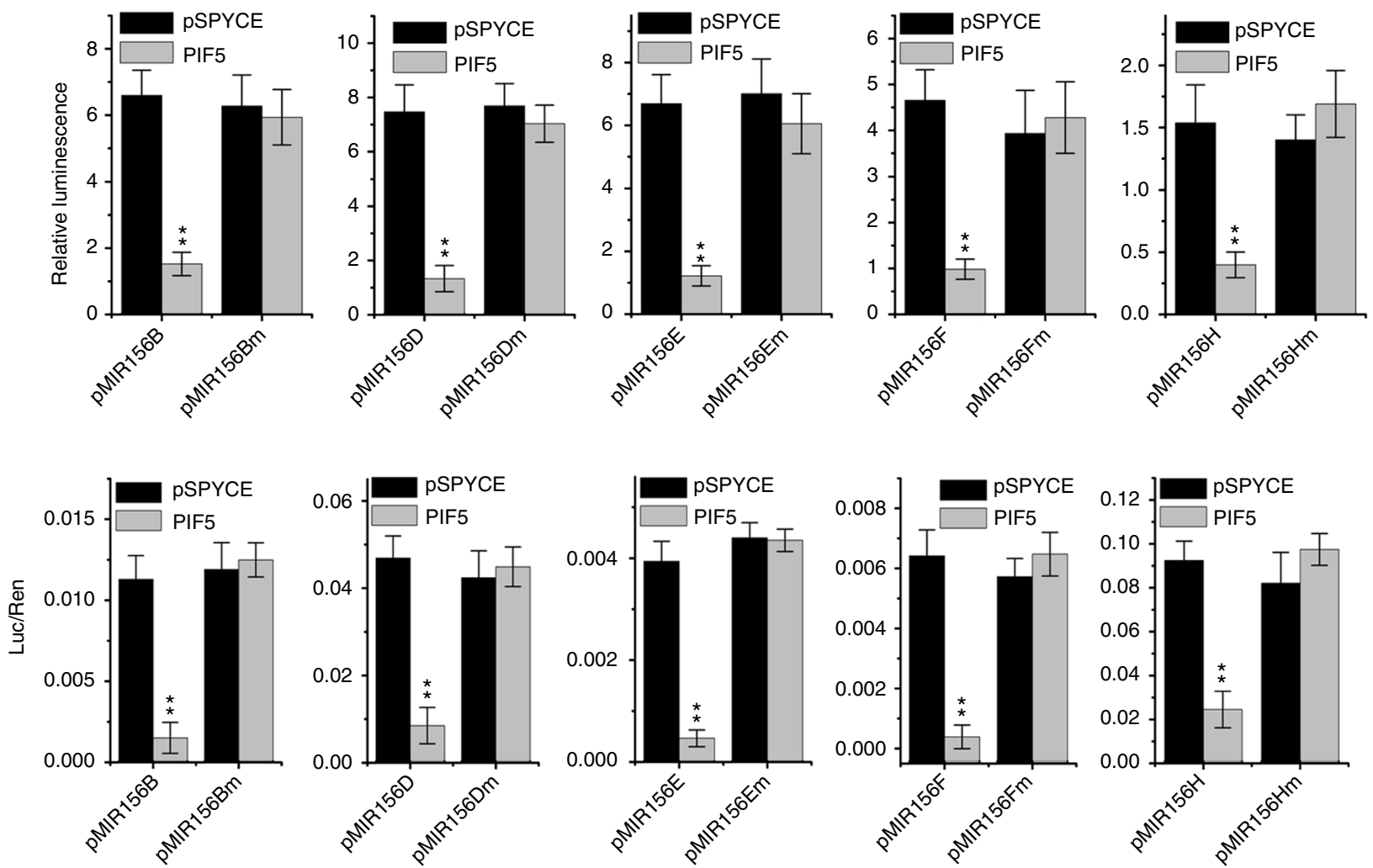

d

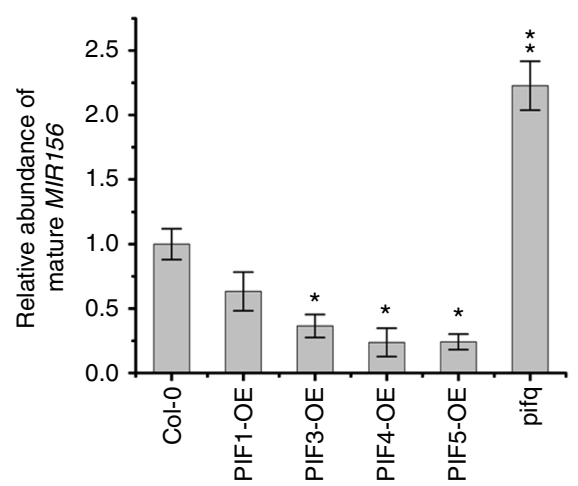

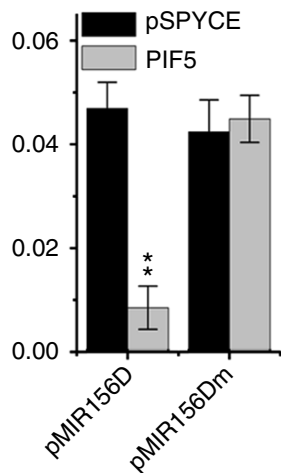
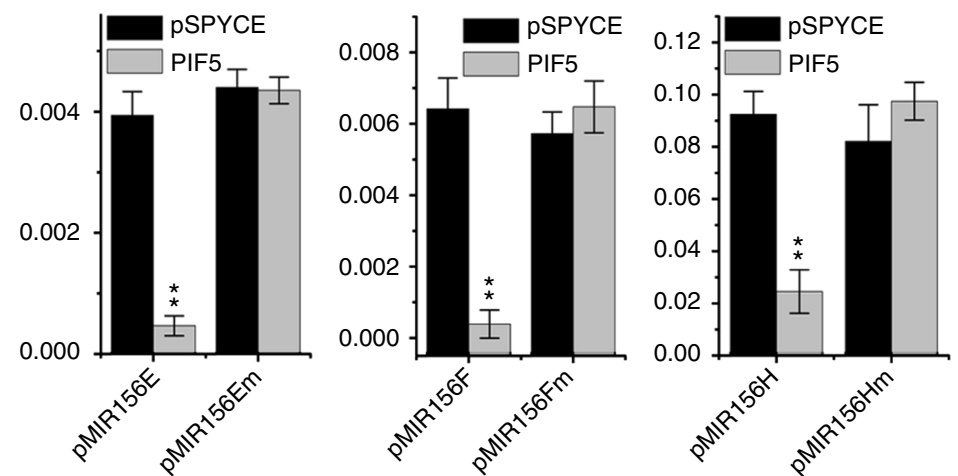
shown in Fig. 3c, PIF5 specifically bound to the promoter fragments containing normal G-box sequence, but not the promoter fragments with mutated G-box sequence. Further, our chromatin immunoprecipitation (ChIP)-PCR assay using the PIF5-HA transgenic plants confirmed enrichment of MIR156B, MIR156D, MIR156E, MIR156F, and MIR156H promoter fragments containing the G-box motif (Fig. 3d). These results verified the in vivo binding of PIF5 to multiple members of the MIR156 gene family and confirmed an earlier report that MIR156E (AT5G11977) was identified as a putative direct target gene of PIF5 ${ }^{13}$.

PIFs directly repress the expression of several MIR156 genes. We next performed a transient expression assay to examine whether PIF proteins could directly regulate the transcription of the target MIR156s. Agrobacterium strains harboring the p35S: PIF effector and PMIR156:LUC reporter plasmids were injected into Nicotiana benthamiana leaf epidermal cells. After incubation in darkness at $25^{\circ} \mathrm{C}$ for $2-3$ days, the pMIR156:LUC reporter gene activity was examined. Co-expression of PIF protein strongly inhibited the $L U C$ reporter gene activities driven by the endogenous promoters of MIR156B, MIR156D, MIR156E, $M I R 156 F$, and $M I R 156 H$, but this inhibition was abolished by mutations in the G-box motifs in these promoters (Fig. $4 a-c$ and Supplementary Fig. 9). Consistent with this, most primary MIR156 transcripts and the total mature MIR156 levels were lower in the 35S::PIF-OE lines, but significantly increased in the pifq plants compared to the WT (Fig. 4d, e). Further, the expression of SPL genes, downstream target genes of miR156, increased in the 35S::PIF-OE lines (Supplementary Fig. 10). We thus concluded that PIF5 (and most likely other PIFs as well) could bind to the promoters of multiple MIR156 members directly and repress their expression.

MIR156s act downstream of PIFs in regulating SAS. To further investigate the possible genetic relationship between PIF and MIR156s in regulating SAS, we generated PIF5-OE/MIR156-OE, pifq/MIR156-OE, PIF5-OE/MIM156, and pifq/MIM156 plants via genetic crosses and examined their responses to simulated shade treatment. As expected, plants of the pifq/MIR156-OE genotype displayed a similar phenotype to that of MIR156-OE line in the number of rosette leaves, plant height, number of rosette-leaf branches, length of the first and second petioles, and the size of the first and second leaves (Fig. 5 and Supplementary Fig. 11). However, PIF5-OE/MIR156-OE displayed fewer rosette leaves and rosette-leaf branches, longer petioles, and elevated plant heights than MIR156-OE, but much more rosette leaves and rosette-leaf branches and shorter petiole length and plant heights than $35 S:$ : PIF5-OE (Fig. 5 and Supplementary Fig. 11). No significant phenotypic differences were detected among theMIM156, PIF5OE/MIM156, and pifq/MIM156 plants (Fig. 5 and Supplementary Fig. 11). These observations, together with the molecular data presented above, support the placement of MIR156 downstream of PIF5 (and most likely other PIFs as well) in regulating various aspects of SAS.

\section{Discussion}

SAS refers to a collection of important adaptive morphological and physiological changes in plants that occur in response to reduced R:FR ratios caused by neighboring vegetation. Although this response is deemed to improve the reproductive success and thus the survival rates of the plants, it is detrimental to agricultural production of most cereal crops. Thus, it is generally believed that SAS has largely been attenuated or refined during crop domestication and genetic improvement ${ }^{8,30}$. Indeed, there is evidence that phytochromes might have been subjected to selection during crop domestication and breeding, and attempts have been made to suppress SAS for better agronomic performance in several crops via manipulating the light signaling pathways through a transgenic approach. However, despite promising, these efforts have made limited success due to the associated pleiotropic negative effects of the transgenes $1,31,32$. As pressure on arable land persists, breeding of high planting density tolerant cultivars will continue to be a major objective in the years ahead. A better understanding of the signaling mechanisms governing SAS will definitely help meet this need.

In this study, we showed that in Arabidopsis thaliana, PIFs, a group of signaling integrators between light and other signals (various hormones, temperature, sugar, and so on $)^{33}$ directly bind to and repress the expression of several MIR156 genes (MIR156B, MIR156D, MIR156E, MIR156F, and MIR156H) in response to simulated shade, causing altered developmental programs in multiple plant organs, and thus plant architecture (including leaf production and expansion, petiole and stem elongation, branches, and flowering time). It is well known that miR156 represents one of the most evolutionarily conserved miRNAs in plants ${ }^{34}$. Among the 17 Arabidopsis SPLs, 11 are miR156 targets, and these SPLs have been shown to regulate diverse developmental processes $^{20,35}$. Based on our results, we propose a putative model in which the FR-inactivated phytochromes (mainly phyB) induce a rapid stabilization and increased accumulation of several PIF proteins (PIF1, PIF3, PIF4, and PIF5), which in turn directly bind to the G-box motifs in the promoters of multiple MIR156 genes and repress their expression, thus alleviating the inhibitory effect of miR156 on the downstream SPL genes. As transcription factors, the activated $S P L$ genes then regulate diverse morphological changes associated with shade-avoidance responses via altering diverse sets of further downstream genes (Fig. 5c). Further studies are required to substantiate this proposition.

Notably, a recent study reported that in Arabidopsis, the transcriptional repressors DELLA proteins physically interact with SPL9 and interfere with its transcriptional activity, causing delayed floral transition by repressing miR172 in leaves and MADS-box genes at shoot apex, thus establishing a link between gibberellin-mediated and miR156/SPL module-mediated flowering pathways ${ }^{36}$. It has also been reported that DELLA proteins can inhibit PIFs proteins (PIF3 and PIF4) by sequestering their DNA-binding domains, thus antagonistically regulating hypocotyl elongation in plants ${ }^{24,37}$. Further, a recent study showed that DELLA proteins can also accelerate degradation of PIF proteins by the $26 \mathrm{~S}$ ubiquitin-proteasome pathway, suggesting a dual regulatory mechanism of PIFs by DELLAs ${ }^{38}$. However, no direct

\footnotetext{
Fig. 4 PIF5 directly represses the expression of MIR156 genes. a Transient expression assays shows that PIF5 directly represses the expression of MIR156B, MIR156D, MIR156E, MIR156F, and MIR156H. Representative images of $N$. benthamiana leaves $72 \mathrm{~h}$ after infiltration were shown. b Quantitative analysis of luminescence intensity in a. Five independent determinations were assessed. Values shown are mean \pm SD ( $n=5$ ). ${ }^{\star \star} P<0.01$ by the Student's $t$-test. c Dual-luciferase assay of pMIR156::LUC expression. The expression of REN was used as an internal control. LUC/REN ration represents the relative activity of the MIR156 promoter. Values given are mean \pm SD $(n=3)$. ${ }^{\star \star} P<0.01$ by the Student's $t$-test. d Transcript levels of mature MIR156 analyzed by qRT-PCR. Eight-day-old WT, PIF overexpressors, and pifq seedlings grown under WL were harvested for RNA extraction. Values given are mean \pm SD ( $n=3$ ). ${ }^{\star} P<$ 0.05 and ${ }^{\star \star} P<0.01$ by the Student's $t$-test. e The relative expression levels of individual primary MIR156 in 8 -day-old WT, $P$ IF overexpression, and pifq seedlings grown under high R:FR conditions. Values given are mean \pm SD $(n=3)$. ${ }^{\star} P<0.05$ and ${ }^{\star \star} P<0.01$ by the Student's $t$-test
} 
a

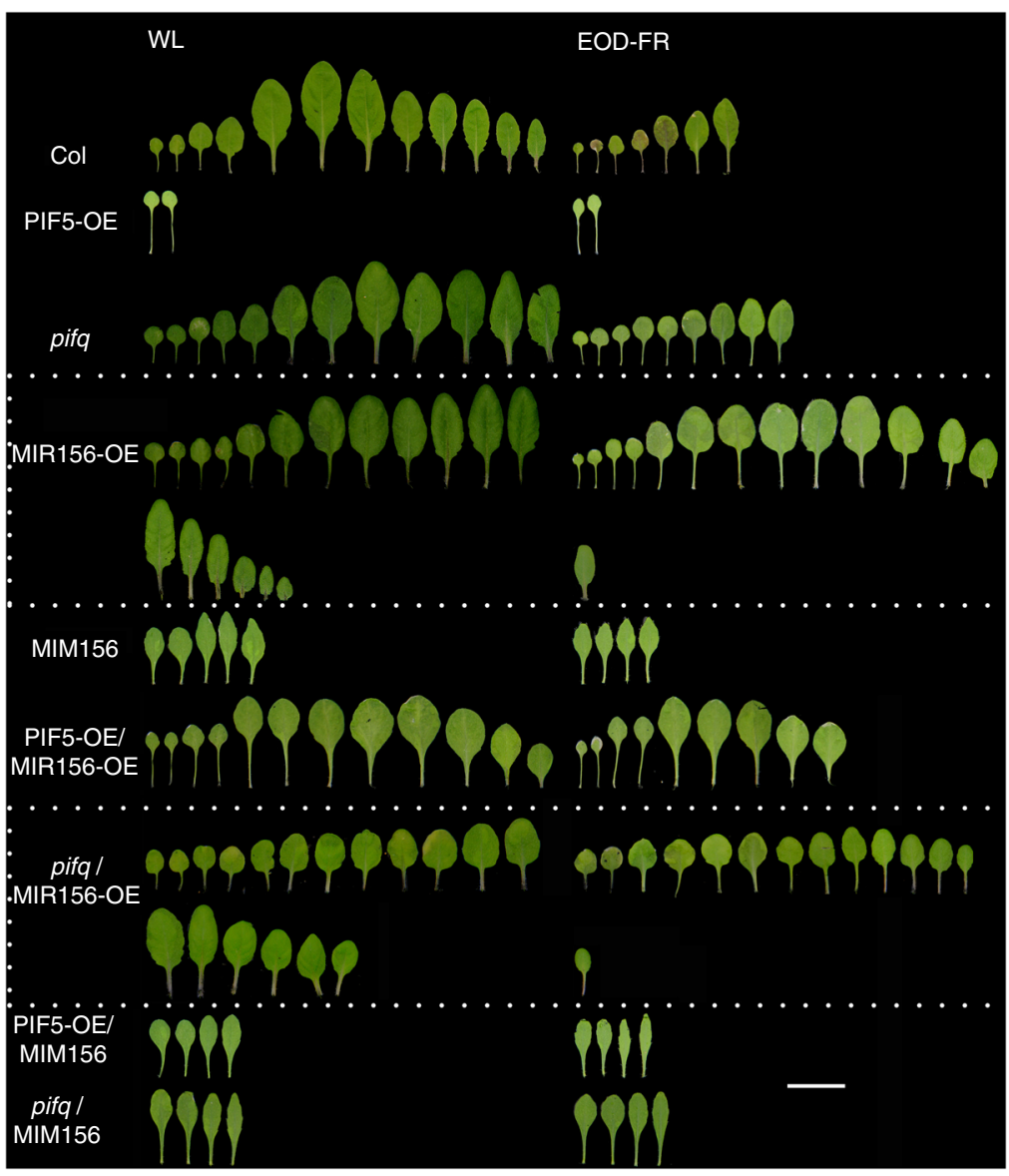

b
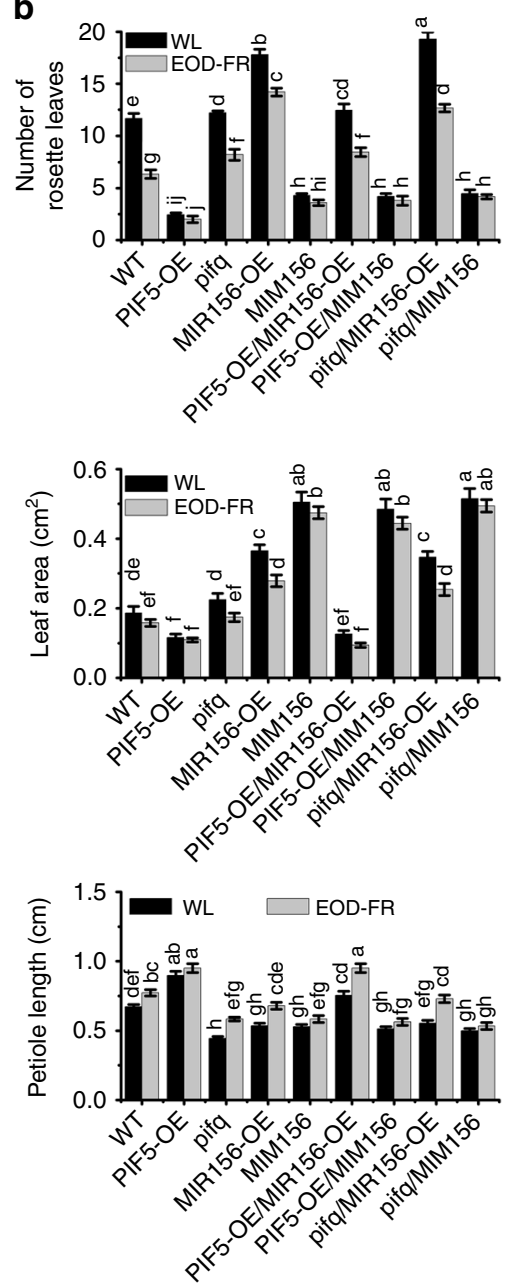

C

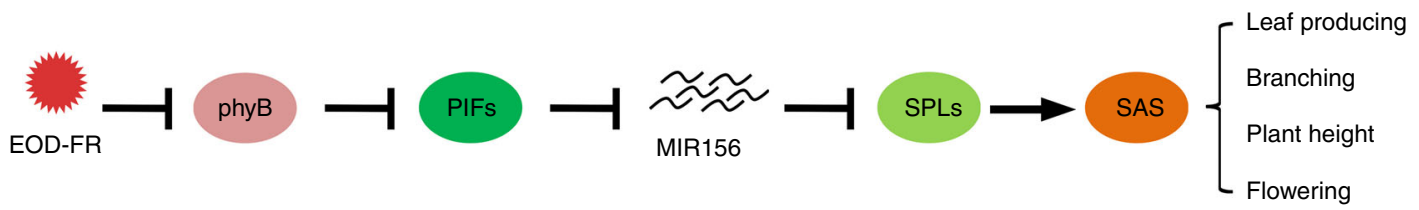

Fig. 5 MIR156s act downstream of PIFs in regulating SAS. a The rosette leaf number, leaf blade area, and petiole length of the first and second leaf of WT, PIF5-OE, pifq, MIR156-OE, MIM156, and their higher order mutants grown under WL with or without EOD-FR treatment. Eight-day-old seedling grown on Murashige and Skoog agar medium under WL were transferred into soil and treated with EOD-FR for 4 weeks before phenotypic analysis. Bar $=2 \mathrm{~cm}$. b Quantification of the rosette leaf number, leaf blade area, and petiole length of WT, PIF5-OE, pifq, MIR156-OE, MIM156, and their higher order mutants grown under WL with or without EOD-FR treatment. Values given are mean \pm SD $(n=12)$. Letters indicate significant differences by two-way ANOVA. c Simplified schematic model depicting the signaling pathway of PIFs and MIR156 to modulate shade-avoidance response in adult Arabidopsis plants. Shade (low R:FR ratios) inactivates phyB and induces a rapid accumulation of PIF proteins. These PIFs then directly bind to the promoters of multiple MIR156 genes and repress their expression, which alleviates the inhibitory effect of MIR156s on their target SPL genes. The activated SPL genes then regulate diverse morphological changes associated with shade-avoidance responses via altering distinct sets of further downstream genes. Arrow: activate; Bar: repress

physical interaction between PIFs (PIF1, PIF3, PIF4, and PIF5) and SPLs (including SPL2, SPL3, SPL4, SPL5, SPL9, SPL10, SPL11, and SPL15) was detected in our yeast two-hybrid assay (Supplementary Fig. 12). Also no direct binding of PIFs to the promoters of above SPLS was detected in our yeast one-hybrid assay (Supplementary Table 2 and Supplementary Fig. 13). These results together suggest that PIFs regulate SPL activity mainly through regulating MIR156 expression.

Accumulating evidence shows that the miR156/SPL regulatory module is highly conserved in land plant species, and plays important roles in regulating diverse plant developmental processes and crop architecture ${ }^{20}$. Examples include the maize TGA1 controlling glume development ${ }^{39}$, maize $L G 1$ controlling leaf and tassel branch angle ${ }^{40,41}$, maize UB2, UB3, and TSH4 regulating tassel and ear development, as well as vegetative tillering $^{42-44}$, rice OsSPL16/GW8 and OsSPL13/GLW7 controlling grain size $^{45,46}$, rice OsSPL14/IPA1/WFP controlling plant architecture $^{47,48}$, rice OsSPL7 and OsSPL17 regulating tillers and panicle architecture ${ }^{49}$, and rice $O s L G 1$ controlling leaf and panicle branch angle 50,51 . Notably, many of these genes are likely targets of selection during crop domestication or breeding for improved plant architecture and agronomic performance. In such 
a context, it will be highly worthy to investigate whether the PHYPIFs-miR156-SPLs regulatory circuitry identified in Arabidopsis also operates in crops, and how the components of this circuitry can be tailored to achieve desirable plant architecture and enhance yield per unit land area.

\section{Methods}

Plant materials and growth conditions. All Arabidopsis materials used in this study are of the Columbia (Col-0) genetic background. The PIF1-OE (35S:::PIF1$M Y C$ ) and PIF3-OE (35S::PIF3-MYC) transgenic lines were kindly provided by Dr. Rongcheng Lin $^{21,22}$. The PIF4-OE (35S::PIF4-HA) and PIF5-OE (35S::PIF5-HA) transgenic lines were supplied by Dr. Jiaqiang Sun ${ }^{23}, 24$. The stock seeds of MIR156-OE (35S::MIR156) ${ }^{25}$ and MIM156 (35S::MIM156) ${ }^{52}$ were purchased from the European Arabidopsis Stock Centre (http://Arabidopsis.info).

Arabidopsis seeds were sown on Murashige and Skoog solid medium containing $1 \%$ sucrose after surface sterilization and vernalized at $4{ }^{\circ} \mathrm{C}$ in the dark for 3 days. Seedlings were then placed in a growth chamber (Percival, USA) and grown under continuous WL conditions at $23^{\circ} \mathrm{C}(16 \mathrm{~h}$ light $/ 8 \mathrm{~h}$ dark) for 8 days before further treatment or transferring into soil.

Tobacco (Nicotiana benthamiana) seeds were directly sown into the soil and grown in the culture room under WL conditions $\left(16 \mathrm{~h} \mathrm{light} / 8 \mathrm{~h}\right.$ dark) at $25^{\circ} \mathrm{C}$ for 1 month before being used for the luciferase activity assay.

EOD-FR treatment. For transcript analysis, 8-day-old seedlings or 3-week-old adult plants grown under continuous WL conditions were treated with FR light $\left(30 \mu \mathrm{mol} \mathrm{m}^{-2} \mathrm{~s}^{-1}\right)$ for $15 \mathrm{~min}$ at the end of the light period (EOD-FR treatment) Seedlings and adult plants were treated for 7 days and harvested at the given time points $(0,0.5,1,3,6 \mathrm{~h})$ after the last treatment and frozen in liquid nitrogen for total RNA extraction.

For phenotypic investigation, 8-day-old seedling were transferred into soil and treated with EOD-FR as described above for 4 weeks until phenotypic investigation.

Phenotypic investigation. For phenotypic analysis, 2-week-old seedlings were grown under normal high R:FR (WL) or simulated shade (EOD-FR) for 4 weeks. After the main inflorescence became visible, the rosette leaves were detached, photographed, and counted. The blade area and petiole length of the first and second leaves were measured by ImageJ software (version 1.38) and averaged for blade area and petiole length, respectively. For rosette-leaf branch number, the rosette-leaf branches (longer than $0.3 \mathrm{~cm}$ ) were counted and normalized to that of WT grown under WL conditions. For plant height, all the plants were measured at the time when growth of 35S:::PIF5-OE plants have ceased. Three biological replicates of each genotype were analyzed.

Promoter analysis. Promoter analysis was conducted using PLACE (http://www. dna.affrc.go.jp/PLACE/signalsacan.html). Promoters of MIR156 genes ( 3 kp upstream of mature MIR156 sequence) were searched for the PBE-box (3'-CACATG-5') or G-box (3'-CACGTG-5') motifs.

Plasmid construction and transformation. For yeast one-hybrid assay, the coding regions of PIF1, PIF3, PIF4, and PIF5 were PCR amplified and ligated to the pJG4-5 vector (Clontech, USA) at the EcoRI restriction site to produce AD-PIFs. Promoter fragment ( $3 \mathrm{~kb}$ upstream of mature MIR156 sequence) of each MIR156 member and SPL member was PCR amplified and ligated to the pLacZi2 $\mu$ vector ${ }^{53}$ digested with SmaI to generate $2 \mu$-pMIR156s and $2 \mu$-pSPLs. For mutagenesis of the G-box or PBE-box in each $2 \mu$-pMIR156 construct, primer pairs were designed according to Agilent Technologies (http://www.genomics.agilent.com) and used to generate plasmid-containing mutant G-box or PBE-box following the manufacturer's instructions.

For yeast two-hybrid assay, the coding regions of PIFs and SPLs were PCR amplified from cDNAs and ligated to the pGADT7 and pGBKT7 vectors (Clontech, USA) at the EcoRI restriction site to generate pGADT7-PIFs and pGBKT7-SPLs, respectively.

To generate the $p M I R 156:: G U S$ constructs, all MIR156 promoters were cloned into the vector pBI101 (Clontech, USA) at the SalI site. All pMIR156::GUS constructs were then transformed into Agrobacterium tumefaciens strain GV3101 and further transformed into WT Arabidopsis (Col-0) using the floral dip $\operatorname{method}^{54}$.

For luciferase assay, the coding region of PIFs was cloned into the SPYCE vector $^{55}$ at the SalI restriction site to produce 35S::PIF-SPYCE. All the MIR156 promoters were amplified from individual WT $2 \mu$-pMIR156s above and then ligated into the plasmid pGreenII0800-LUC (Biovector, USA) digested with SalI to produce pMIR156s::LUC. The MIR156 promoters harboring mutant G-box or PBEbox were generated using the corresponding same primer pairs with mutant $2 \mu$ pMIR156s plasmids above as templates and inserted into the pGreenII0800-LUC vector at the SalI site. The 35S:::PIF-SPYCE or PMIR156::LUC constructs were introduced into Agrobacterium tumefaciens strain EHA105 and these EHA105 cells harboring 35::PIF5-SPYCE or pMIR156::LUC were co-injected into Nicotiana benthamiana leaves.

Table 1 .

RNA extraction and quantitative RT-PCR. Total RNA was isolated with Trizol reagent (Invitrogen, USA). For quantitative detection of the primary MIR156s, cDNA was firstly synthesized using M-MLV (Promega, USA) and detected with the TransStart Tip Green qPCR Super Mix (TransGen Biotech, China). For comparison of mature MIR156, total RNA was purified with the miRcute miRNA purification kit (Tiangen Biotech, China) and further cDNA was reverse transcribed with the miRcute miRNA first-strand cDNA synthesis kit (Tiangen Biotech, China). The mature miR156 was detected with the miRcute miRNA qPCR Detection Kit (Tiangen Biotech, China). Quantitative RT-PCR was conducted with the SYBR Premix ExTaq kit (Takara, Japan) in a total volume of $25 \mu \mathrm{L}$ on the Applied Biosystems 7500 real-time PCR system according to the manufacturer's manual. The level of PP2A (AT1G13320) transcript was adopted as an internal control. The expression of mature MIR156 or each primary MIR156 (priMIR156A H) in different samples was calculated by the $2^{\Delta \mathrm{Ct}}$ method, in which: $\Delta \mathrm{Ct}=\mathrm{Ct}($ PP2A $)-\mathrm{Ct}($ target $)$. The expression level of target gene after treatments are the ratio of expression in treated samples compared with the controls by the $2^{\Delta \Delta \mathrm{Ct}}$ method, in which $\Delta \Delta \mathrm{Ct}=\left(\mathrm{Ct}_{\mathrm{PP} 2 \mathrm{~A}}-\mathrm{Ct}_{\text {target }}\right)$ treatment $-\left(\mathrm{Ct}_{\mathrm{PP} 2 \mathrm{~A}}-\mathrm{Ct}_{\text {target }}\right)$ control.

All the primers used for qRT-PCR above are shown in Supplementary Table 1.

Yeast one-hybrid and two-hybrid assay. To test the binding of PIF to MIR156 or $S P L$ promoter in yeast, the plasmids $A D$-PIFs and $2 \mu$-pMIR156 (or $2 \mu$-pSPL) were co-transformed into the yeast strain EGY48 mediated by PEG4000. The positive transformants grown on the $\mathrm{SD} /$-Trp/-Ura medium (Clontech, USA) were transferred to the selection medium containing raffinose, galactose, and 5-Bromo-4chloro-3-indolyl-beta-D-galactopyranoside (Amresco, USA) for blue color development. To quantify the $\beta$-galactosidase activity, the positive clones were cultured in liquid SD/-Trp/-Ura medium overnight, re-suspended in Z-buffer $(40 \mathrm{mM}$ $\mathrm{NaH}_{2} \mathrm{PO}_{4}, 60 \mathrm{mM} \mathrm{Na} \mathrm{HPO}_{4}, 1 \mathrm{mM} \mathrm{MgSO}{ }_{4}, 10 \mathrm{mM} \mathrm{KCl}, \mathrm{pH}$ 7.0, $50 \mathrm{mM} \beta$-mercaptoethanol), and then lysed with chloroform and $0.1 \%$ sodium dodecyl sulfate. Then 2-Nitrophenyl- $\beta$-D-galactopyranoside was added into the supernatants and incubated together at $28^{\circ} \mathrm{C}$ for $5 \mathrm{~min}$. The reaction was terminated by addition of $2 \mathrm{M} \mathrm{Na}_{2} \mathrm{CO}_{3}$ and the optical density of the supernatant was measured.

To examine possible interaction between PIF and SPL proteins, the plasmids pGADT7-PIFs and pGBKT7-SPLs were co-transformed into the yeast strain AH109 mediated by PEG4000. The positive transformants were first selected on the SD/-Ura/-Leu medium (Clontech, USA) and then transferred to SD/-Ura/Leu/-His/-Ade selection medium (Clontech, USA) to detect the interaction. The interaction between pGADT7-T and pGBKT7-53 was used as a positive control.

Recombinant protein production. The truncated fragment of PIF5 encoding the bHLH domain (52 aa, $\mathrm{Glu}_{259}$ - $\mathrm{Gln}_{310}$ ) was PCR amplified using the plasmid ADPIF5 as the template and cloned into the vector GEX-4T-1 (Amersham, USA) at the EcoRI site to generate the pGEX-4T-PIF5 construct. The plasmid was transformed into the Escherichia coli Rosetta (DE3) strain. Expression of PIF5-GST fusion protein was induced by $0.4 \mathrm{mM}$ isopropyl $\beta$-D-thiogalactopyranoside and incubation at $16^{\circ} \mathrm{C}$ overnight. Cells were spinned down at $5000 \mathrm{rpm}$ for $10 \mathrm{~min}$ and lysed by sonication. After centrifugation at $13,000 \mathrm{rpm}$ for $40 \mathrm{~min}$ at $4{ }^{\circ} \mathrm{C}$, the PIF5-GST fusion protein from supernatant was purified using glutathionesepharose resin (GE Healthcare, USA). The eluted PIF5-GST fusion protein was dialyzed against the dialysis buffer $(10 \mathrm{mM}$ Tris-Cl, $\mathrm{pH} 8.0)$, aliquoted and stored at $-80^{\circ} \mathrm{C}$ until use.

Gel mobility shift assay. For DNA EMSA of PIF5 binding to promoters of MIR156E or MIR156F, two complementary 60-bp length oligonucleotides containing the G-box of MIR156E or MIR156F were synthesized and labeled with biotin separately. DNA probes were obtained by annealing two complementary oligonucleotides. DNA gel mobility shift assay was performed using the EMSA kit (Beyotime, China) following the manufacturer's protocol. Briefly, biotin-labeled probes were incubated for $20 \mathrm{~min}$ with the GST or GST-PIF5 protein in the binding buffer at room temperature. For competition reaction, $50 \times$ and $100 \times$ unlabeled cold probes were mixed with the labeled probes. The DNA-protein complex was separated by $5 \%$ native polyacrylamide gel electrophoresis and the signal of biotin was developed using the Biostep Celvin S420 system (Biostep, German).

ChIP-PCR assay. For ChIP-PCR assay, 8-day-old seedlings harboring 35S:PIF5$H A$ were treated with EOD-FR for one time and kept in darkness for $3 \mathrm{~h}$. Then the seedlings were cross-linked with $1 \%$ formaldehyde and the chromatin complexes were sonicated. The immuno complex was prepared following the method of Guo et $\mathrm{al}^{56}$. Briefly, after centrifugation at $14,000 \times g$ for $30 \mathrm{~min}$, the supernatant was pre-cleared with Protein-A-Agarose (Santa Cruz Bio, USA) and incubated at $4{ }^{\circ} \mathrm{C}$ for $1 \mathrm{~h}$. After spinning, the supernatant was moved into a microtube and the HAspecific antibodies (Cali-Bio, USA) were added (for negative control, no antibodies were added). After incubation at $4^{\circ} \mathrm{C}$ for $3 \mathrm{~h}$, the Protein-A-Agarose was added and incubated for $2 \mathrm{~h}$. After washing with $1 \mathrm{~mL}$ of each of the following buffers: 
Buffer A (50 mM HEPES-KOH, pH 7.5, 1 mM EDTA, 140 mM NaCl, $0.1 \%$ Nadeoxycholate, $1 \%$ Triton X-100, 1 mM PMSF), Buffer B (50 mM HEPES-KOH, pH 7.5, $1 \mathrm{mM}$ EDTA, $500 \mathrm{mM} \mathrm{NaCl}, 0.1 \%$ NaDeoxycholate, $1 \%$ Triton X-100, 1 mM PMSF), Buffer C (10 mM Tris-HCl, pH 8.0, 0.5\% NP-40, 0.5\% NaDeoxycholate, $250 \mathrm{mM} \mathrm{LiCl}, 1 \mathrm{mM}$ EDTA), and Buffer D (10 mM Tris-HCl, $\mathrm{pH} \mathrm{8.0,} 1$ mM EDTA), the immuno complex was eluted from the agarose beads. The precipitated DNA was then recovered and quantified using quantitative PCR with their individual primer pairs. The values were standardized to the input DNA to obtain the enrichment fold. PP2A was used as an internal control.

Luciferase activity assay. $N$. benthamiana leaves were co-injected with $35 S$ :: PIF5SPYCE and PMIR156::LUC and incubated at $25^{\circ} \mathrm{C}$ for $2-3$ days. To image the luciferase luminescence, the leaves were detached and sprayed with $20 \mathrm{mg} \mathrm{mL}^{-1}$ potassium luciferin (Gold Biotech, USA) and incubated in darkness for $5 \mathrm{~min}$. The luciferase luminescence from the infiltrated area was imaged using Night SHADE LB 985 system (Berthold, Germany) with a $30 \mathrm{~s}$ exposure time, $4 \times 4$ binning, slow readout, and high gain. Quantification of luciferase activity was carried out with IndiGO software (version 2.03.0) using average luminescence counts per second Luciferase activity was also measured using the Dual-Luciferase Reporter Assay System (Promega, USA) following the manufacturer's protocol and the relative firefly luciferase activity was counted as the ratio of firefly to Renilla luciferase activity (LUC/REN) for each sample.

GUS assay. Eight-day-old pMIR156::GUS seedlings were submerged, evacuated, and kept in the GUS staining solution $\left(2 \mathrm{mM} \mathrm{K}_{4} \mathrm{Fe}(\mathrm{CN})_{6}, 2 \mathrm{mM} \mathrm{K}_{3} \mathrm{Fe}(\mathrm{CN})_{6}, 1 \mathrm{mg}\right.$ $\mathrm{mL}^{-1} \mathrm{x}$-Gluc, $10 \mathrm{mM}$ EDTA, $0.1 \%$ Triton X-100, $100 \mathrm{mg} \mathrm{mL}^{-1}$ chloramphenicol, $50 \mathrm{mM} \mathrm{Na} 2 \mathrm{HPO}_{4}-\mathrm{NaH}_{2} \mathrm{PO}_{4}, \mathrm{pH} 7.0$ ) at $37^{\circ} \mathrm{C}$ for $12 \mathrm{~h}$. Then the stained seedlings were decolorized and fixed in 70\% (v/v) ethanol and photographed using a Zeiss dissecting microscope. GUS activity assay was performed following the method of Jefferson et $\mathrm{al}^{57}$.

Western blotting. Eight-day-old PIF overexpression seedlings (35S::PIF1-MYC, 35S::PIF3-MYC, 35S:::PIF4-HA, and 35S::PIF5-HA) grown under WL or treated with EOD-FR for one time were harvested at $0,0.5,1,3,6 \mathrm{~h}$. Protein extracts were prepared as describe in Al-Sady et al. ${ }^{58}$ and total protein was quantified with the Quick Start Bradford Protein Assay Kit (Bio-Rad, USA). The purified anti-MYC antibodies (M047-7, MBL, Japan) or anti-HA antibodies (561-7, MBL, Japan) with 1:2000 and 1:10000 dilutions, respectively, were used to detect PIF1 and PIF3, or PIF4 and PIF5, respectively. As an internal control, the $\beta$-tubulin monoclonal antibodies (As10680, Agrisera, Sweden) at a dilution of 1:1000 were used with the goat anti-rabbit secondary antibodies (458, MBL, Japan) at a dilution of 1:8000. The original blot images were provided in Supplementary Fig. 14.

Statistical analysis. All real-time PCR reactions and other quantitative analysis were repeated at least three times. To evaluate the significant differences among the various genotypes treated or untreated with EOD-FR, the method of Brady et al. ${ }^{59}$ about two-way analysis of variance (ANOVA) with interaction was adopted by using the aov functions implemented in the "stats" package of the R programming language (version 3.3.1). Also the TukeyHSD in the "stats" package was used for all pairwise comparisons, with $p$-values corrected for multiple comparisons to control against type I errors. The ANOVA results were shown in Supplementary Data 1. The Student's $t$-test was adopted to analyze the significant differences between two groups.

Data availability. All relevant data that support the findings of this study are included within the article or available from the authors upon request.

Received: 2 February 2017 Accepted: 24 June 2017

Published online: 24 August 2017

\section{References}

1. Warnasooriya, S. N. \& Brutnell, T. P. Enhancing the productivity of grasses under high-density planting by engineering light responses: from model systems to feed stocks. J. Exp. Bot. 65, 2825-2834 (2014).

2. Duvick, D. N. in What is Yield? Developing Drought and Low N-Tolerant Maize (eds Edmeades, G. O., Bänziger, B., Mickelson, H. R. \& Pena-Valdivia, C. B.) pp 332-335 (CIMMYT, 1997).

3. Casal, J. J. Shade avoidance. Arabidopsis Book 10, e0157 (2012).

4. Smith, H. \& Whitelam, G. C. The shade avoidance syndrome: multiple responses mediated by multiple phytochromes. Plant Cell Environ. 20, 840-844 (1997).

5. Weinig, C., Johnston, J., German, Z. M. \& Demink, L. M. Local and global costs of adaptive plasticity to density in Arabidopsis thaliana. Am. Nat. 167, 826-836 (2006).

6. Kebrom, T. H. \& Brutnell, T. P. The molecular analysis of the shade avoidance syndrome in the grasses has begun. J. Exp. Bot. 58, 3079-3089 (2007).
7. Ballaré, C. L. Light regulation of plant defense. Annu. Rev. Plant Biol. 65 335-363 (2014)

8. Smith, H. Ecology of photomorphogenesis: clues to a transgenic programme of crop plant improvement. Photochem. Photobiol. 56, 815-822 (1992).

9. Devlin, P. F. et al. Phytochrome D acts in the shade-avoidance syndrome in Arabidopsis controlling elongation growth and flowering time. Plant Physiol. 119, 909-915 (1999).

10. Reed, J. W., Nagpal, P., Poole, D. S., Furuya, M. \& Chory, J. Mutations in the gene for the red/far-red light receptor phytochrome B alter cell elongation and physiological responses throughout Arabidopsis development. Plant Cell 5, 147-157 (1993).

11. Franklin, K. A. Shade avoidance. New Phytol. 179, 930-944 (2008).

12. Lorrain, S., Allen, T., Duek, P. D., Whitelam, G. C. \& Fankhauser, C. Phytochrome-mediated inhibition of shade avoidance involves degradation of growth-promoting bHLH transcription factors. Plant J. 53, 312-323 (2008).

13. Hornitschek, P. et al. Phytochrome interacting factors 4 and 5 control seedling growth in changing light conditions by directly controlling auxin signaling. Plant J. 71, 699-711 (2012).

14. Leivar, P. et al. Dynamic antagonism between phytochromes and PIF family basic helix-loop-helix factors induces selective reciprocal responses to light and shade in a rapidly responsive transcriptional network in Arabidopsis. Plant Cell 24, 1398-1419 (2012).

15. Li, L. et al. Linking photoreceptor excitation to changes in plant architecture. Genes Dev. 26, 785-790 (2012).

16. Roig-Villanova, I. \& Martínez-García, J. F. Plant responses to vegetation proximity: a whole life avoiding shade. Front. Plant Sci. doi:10.3389/ fpls.2016.00236 (2016).

17. Tao, Y. et al. Rapid synthesis of auxin via a new tryptophan-dependent pathway is required for shade avoidance in plants. Cell 133, 164-176 (2008).

18. Nozue, K. et al. Shade avoidance components and pathways in adult plants revealed by phenotypic profiling. PLoS Genet. 11, e1004953 (2015).

19. Huijser, P. \& Schmid, M. The control of developmental phase transitions in plants. Development 138, 4117-4129 (2011).

20. Wang, H. \& Wang, H. The miR156/SPL module, a regulatory hub and versatile toolbox, gears up crops for enhanced agronomic traits. Mol. Plant 8, 677-688 (2015).

21. Moon, J., Zhu, L., Shen, H. \& Huq, E. PIF1 directly and indirectly regulates chlorophyll biosynthesis to optimize the greening process in Arabidopsis. Proc. Natl. Acad. Sci. USA 105, 9433-9438 (2008).

22. Chen, D. et al. Antagonistic basic helix-loop-helix/bZIP transcription factors form transcriptional modules that integrate light and reactive oxygen species signaling in Arabidopsis. Plant Cell 25, 1657-1673 (2013).

23. Nozue, K. et al. Rhythmic growth explained by coincidence between internal and external cues. Nature 448, 358-361 (2007).

24. de Lucas, M. et al. A molecular framework for light and gibberellin control of cell elongation. Nature 451, 480-484 (2008).

25. Schwab, R. et al. Specific effects of microRNAs on the plant transcriptome. Dev. Cell 8, 517-527 (2005).

26. Wei, S. et al. Arabidopsis mutant sk156 reveals complex regulation of SPL15 in a miR156-controlled gene network. BMC Plant Biol. doi:10.1186/1471-2229-12169 (2012)

27. Lei, K. J., Lin, Y. M. \& An, G. Y. miR156 modulates rhizosphere acidification in response to phosphate limitation in Arabidopsis. J. Plant Res. 129, 275-284 (2016).

28. Leivar, P. \& Quail, P. H. PIFs: pivotal components in a cellular signaling hub. Trends Plant Sci. 16, 19-28 (2011).

29. Ciolfi, A. et al. Dynamics of the shade-avoidance response in Arabidopsis. Plant Physiol. 163, 331-353 (2013).

30. Maddonni, G. A., Otegui, M. E., Andrieu, B., Chelle, M. \& Casal, J. J. Maize leaves turn away from neighbors. Plant Physiol. 130, 1181-1189 (2002).

31. Sawers, R. J., Sheehan, M. J. \& Brutnell, T. P. Cereal phytochromes: targets of selection, targets for manipulation? Trends Plant Sci. 10, 138-143 (2005).

32. Chen, K. \& Gao, C. Targeted genome modification technologies and their applications in crop improvements. Plant Cell Rep. 33, 575-583 (2014).

33. Leivar, P. \& Monte, E. PIFs: systems integrators in plant development. Plant Cell 26, 56-78 (2014).

34. Axtell, M. J. \& Bowman, J. L. Evolution of plant microRNAs and their targets. Trends Plant Sci. 13, 343-349 (2008).

35. Wu, G. \& Poethig, R. S. Temporal regulation of shoot development in Arabidopsis thaliana by miR156 and its target SPL3. Development 133, 3539-3547 (2006).

36. Yu, S. et al. Gibberellin regulates the Arabidopsis floral transition through miR156-targeted SQUAMOSA promoter binding-like transcription factors. Plant Cell 24, 3320-3332 (2012).

37. Feng, S. et al. Coordinated regulation of Arabidopsis thaliana development by light and gibberellins. Nature 451, 475-479 (2008).

38. Li, K. et al. DELLA-mediated PIF degradation contributes to coordination of light and gibberellin signalling in Arabidopsis. Nat. Commun. 7, 11868 (2016). 
39. Dorweiler, J. \& Doebley, J. Developmental analysis of teosinte glume architecturel: a key locus in the evolution of maize (Poaceae). Am. J. Bot. 84, 1313-1322 (1997)

40. Moreno, M. A., Harper, L. C., Krueger, R. W., Dellaporta, S. L. \& Freeling, M. Liguleless 1 encodes a nuclear-localized protein required for induction of ligules and auricles during maize leaf organogenesis. Genes Dev. 11, 616-628 (1997).

41. Bai, F., Reinheimer, R., Durantini, D., Kellogg, E. A. \& Schmidt, R. J. TCP transcription factor, branch angle defective 1 (BAD1), is required for normal tassel branch angle formation in maize. Proc. Natl Acad. Sci. USA 109 12225-12230 (2012)

42. Wang, H. et al. The origin of the naked grains of maize. Nature 436, 714-719 (2005).

43. Studer, A., Zhao, Q., Ross-Ibarra, J. \& Doebley, J. Identification of a functional transposon insertion in the maize domestication gene tbl. Nat. Genet. 43, 1160-1163 (2011).

44. Chuck, G. S., Brown, P. J., Meeley, R. \& Hake, S. Maize SBP-box transcription factors unbranched 2 and unbranched 3 affect yield traits by regulating the rate of lateral primordia initiation. Proc. Natl Acad. Sci. USA 111, 18775-18780 (2014).

45. Wang, S. et al. Control of grain size, shape and quality by OsSPL16 in rice. Nat. Genet. 44, 950-954 (2012).

46. $\mathrm{Si}$, L. et al. OsSPL13 controls grain size in cultivated rice. Nat. Genet. 48, 447-456 (2016).

47. Jiao, Y. et al. Regulation of OsSPL14 by OsmiR156 defines ideal plant architecture in rice. Nat. Genet. 42, 541-544 (2010).

48. Miura, K. et al. OsSPL14 promotes panicle branching and higher grain productivity in rice. Nat. Genet. 42, 545-549 (2010).

49. Wang, L. et al. Coordinated regulation of vegetative and reproductive branching in rice. Proc. Natl Acad. Sci. USA 112, 15504-15509 (2015).

50. Lee, J., Park, J. J., Kim, S. L., Yim, J. \& An, G. Mutations in the rice Ligulelessgene result in a complete loss of the auricle, ligule, and laminar joint. Plant Mol. Biol. 65, 487-499 (2007).

51. Ishii, T. et al. OsLG1 regulates a closed panicle trait in domesticated rice. Nat. Genet. 45, 462-465 (2013)

52. Franco-Zorrilla, J. M. et al. Target mimicry provides a new mechanism for regulation of microRNA activity. Nat. Genet. 39, 1033-1037 (2007).

53. Lin, R. et al. Transposase-derived transcription factors regulate light signaling in Arabidopsis. Science 318, 1302-1305 (2007).

54. Clough, S. J. \& Bent, A. F. Floral dip: a simplified method for Agrobacteriummediated transformation of Arabidopsis thaliana. Plant J. 16, 735-743 (1998).

55 . Walter, M. et al. Visualization of protein interactions in living plant cells using bimolecular fluorescence complementation. Plant J. 40, 428-438 (2004).

56. Guo, L. et al. Isolation of heat shock factor HsfAla-binding sites in vivo revealed variations of heat shock elements in Arabidopsis thaliana. Plant Cell Physiol. 49, 1306-1315 (2008).

57. Jefferson, R. A., Kavanagh, T. A. \& Bevan, M. W. GUS fusions: betaglucuronidase as a sensitive and versatile gene fusion marker in higher plants. EMBO J. 6, 3901-3907 (1987).
58. Al-Sady, B., Ni, W., Kircher, S., Schäfer, E. \& Quail, P. H. Photo activated phytochrome induces rapid PIF3 phosphorylation prior to proteasome mediated degradation. Mol. Cell 23, 439-446 (2006).

59. Brady, S. M. et al. Reassess the $t$ test: interact with all your data via ANOVA. Plant Cell 27, 2088-2094 (2015).

\section{Acknowledgements}

We thank Dr. Rongcheng Lin (Institute of Botany, Chinese Academy of Sciences) for kindly providing the transgenic seeds of 35S::PIF1-MYC and 35S::PIF3-MYC; Dr. Jiaqiang Sun (Institute of Crop Science, Chinese Academy of Agricultural Sciences) for providing the transgenic seeds of 35S::PIF4-HA and 35S::PIF5-HA. This work was supported by grants from National Natural Science Foundation of China (31570191) and Beijing Natural Science Foundation (5162024).

\section{Author contributions}

H.W. and Y.X. designed the research; Y.X., Y.L., X.M., B.W., G.W., and H.W. performed the research and analyzed the data; H.W. and Y.X. wrote the paper.

\section{Additional information}

Supplementary Information accompanies this paper at doi:10.1038/s41467-017-00404-y.

Competing interests: The authors declare no competing financial interests.

Reprints and permission information is available online at http://npg.nature.com/ reprintsandpermissions/

Publisher's note: Springer Nature remains neutral with regard to jurisdictional claims in published maps and institutional affiliations.

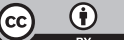

Open Access This article is licensed under a Creative Commons Attribution 4.0 International License, which permits use, sharing, adaptation, distribution and reproduction in any medium or format, as long as you give appropriate credit to the original author(s) and the source, provide a link to the Creative Commons license, and indicate if changes were made. The images or other third party material in this article are included in the article's Creative Commons license, unless indicated otherwise in a credit line to the material. If material is not included in the article's Creative Commons license and your intended use is not permitted by statutory regulation or exceeds the permitted use, you will need to obtain permission directly from the copyright holder. To view a copy of this license, visit http://creativecommons.org/ licenses/by/4.0/.

(C) The Author(s) 2017 